\title{
OSTI
}

\section{Measurement of Input Coupler Matching of a Loaded Storage Ring Single-Cell Cavity}

\author{
Jin Wook Cho, Yoon Kang \\ Advanced Photon Source \\ Argonne National Laboratory \\ 9700 S. Cass Ave., Argonne, IL 60439
}

August 14, 1995

\section{Introduction}

In the APS storage ring cavities, magnetic loop type input couplers are used. The loaded $Q$ fo a cavity varies as the beam loading changes ${ }^{1}$. The beam loading changes the cavity input impedance. Therefore, the input coupler must be adjusted to maintain a good impedance match.

Measurements have been made to determine the coupler loop position (depth of penetration) with respect to various loading conditions in a storage ring single-cell cavity. An input coupler was inserted into the storage ring single-cell cavity at various loaded $\mathrm{Q}$ points, then matched. The relationship between the coupling coefficient, $\beta$, and the gap width, where gap width is the separation between the input coupler flange and the cavity port flange (Fig. 1), was studied. A coupling coefficient is defined ${ }^{2}$ as

$$
\beta=\frac{Q_{O}}{Q_{L}}-1
$$

where $Q_{O}$ is the unloaded $Q$ and $Q_{L}$ is the loaded $Q$, repectively. Depending on the extent of deQing of the fundamental mode in the single-cell cavity; the input coupler was positioned further in or out of the cavity accordingly to achieve matching at $50 \Omega$. Damping the cavity simulates the beam loading when the beam passes through the single-cell cavities.

\section{Test Setup}

As shown in the diagram (Fig. 2), the basic apparatus consisted of a stainless prototype cavity - which is identical in dimensions to the storage ring single-cell cavity - a network analyzer(HP $8510 \mathrm{C}$ ), a pair of field probes, an H-loop damper, and an input coupler. The input coupler (Fig. 3 ) was placed on the top port of the cavity for easier vertical positioning, and the field probes were positioned on the side ports, as described in section 2 below. As for vertical positioning 


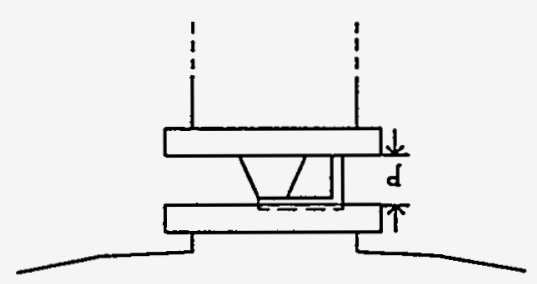

Figure 1: Input Coupler Setup

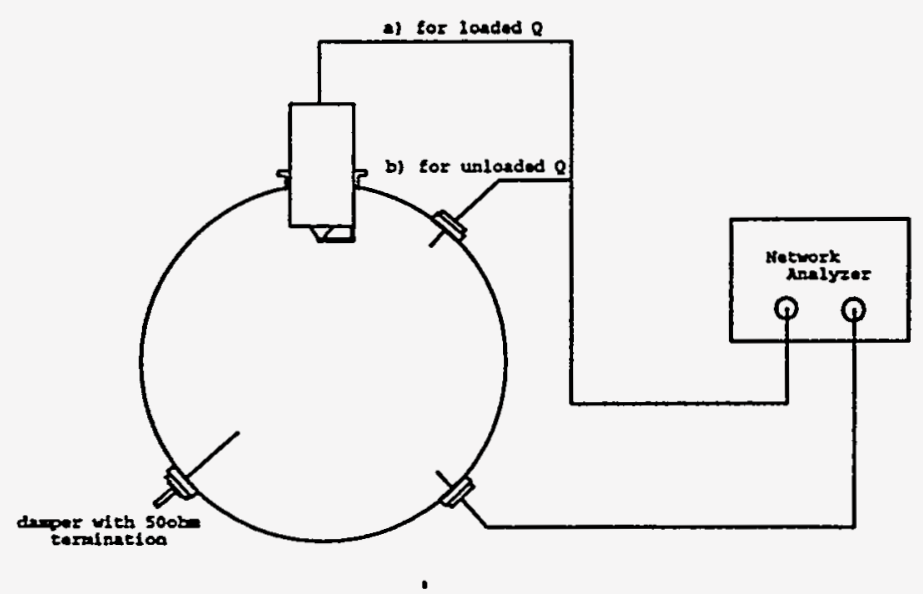

Figure 2: Experiment Setup

of the input coupler, four aluminum tubes were cut out at $\frac{1}{2}, \frac{3}{4}, 1$, and $1 \frac{1}{4}$ inches long, and the finer adjustments were made adding copper rings, which were about $\frac{1}{16}$ inches thick each. For unloaded-Q measurements, two weakly coupled field probes were used, while one field probe was used along with the input coupler for the loaded-Q measurements.

\subsection{Damper}

The damper (Fig. 4) is a simple loop made of a high voltage copper wire to damp the azimuthal magnetic field of the $\mathrm{TM}_{01}$ fundamental mode. One end of the wire is connected to a center conductor of a coaxial connector, while the other end is shorted to the outer conductor by being bolted to the copper plate where the connector is mounted. Bolting allowed easy adjustment of the loop size of the damper, thus changing the $\mathrm{Q}$ value of the cavity. Finally, the coaxial connector is terminated with a $50 \Omega$ load. The damper was positioned at one of the side ports of the cavity.

\subsection{Field Probes}

The field probes (Fig. 5) were made much like the damper, except the loop sizes were made much smaller to minimize the deQing done on the fundamental mode. They were positioned 90 degrees apart radially on the center plane of the cavity. 


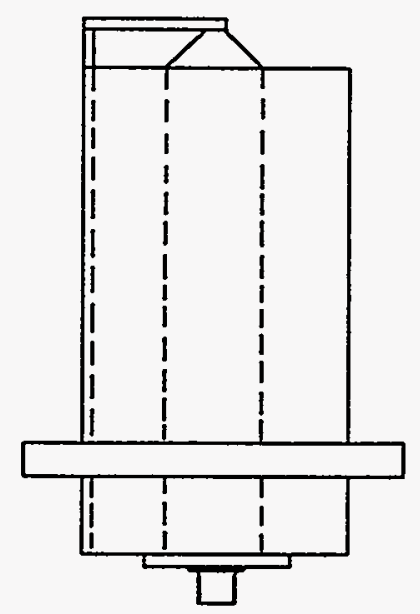

Figure 3: Input Coupler

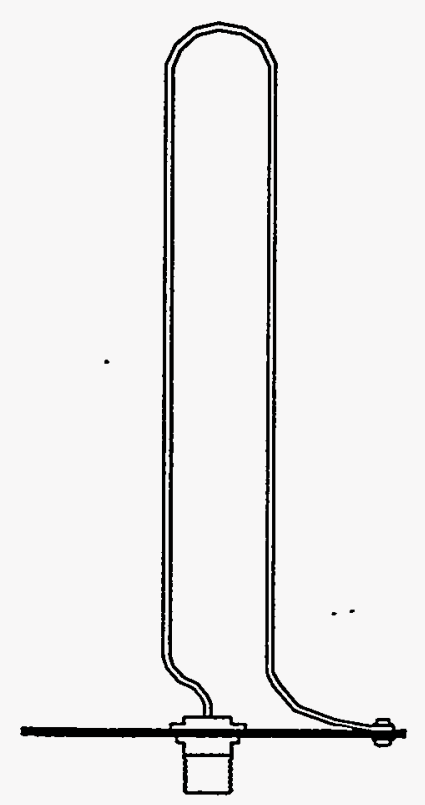

Figure 4: Damper

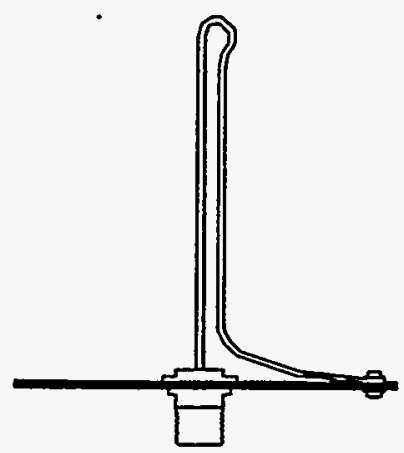

Figure 5: Field Probes 


\section{Measurements}

\subsection{Preliminary Test Results}

In order to determine the basic trend of relationships between the coupling coefficient and the distance from the face of the cavity (in inches), $d$, a preliminary test was done at $\beta=1,2.5$, and 4.5.

\begin{tabular}{|c|c|c|c|c|}
\hline Frequency & Impedance(s11) & Q & Coupl.Coeff. & d \\
\hline 352.316 & $50.56+\mathrm{j} .37$ & 9100 & 1.176 & 1.02 \\
352.333 & $49.2-\mathrm{j} .48$ & 5600 & 2.536 & 0.75 \\
352.311 & $50.18+\mathrm{j} .05$ & 3550 & 4.577 & 0.424 \\
\hline
\end{tabular}

\subsection{Final Test Results}

The final test was performed with nine different loaded $Q$ points. In order to ensure accuracy, calibrations were done for each set of cables: one for the unloaded $Q$ and the other for the loaded $\mathrm{Q}$ measurement since one of the cables used was about 4 feet longer than the one used for the unloaded $Q$ measurement. The data gathered (see table) followed the trend found in the preliminary test, and the relation seemed linear with slight variance. The data are plotted in Figure 6.

\begin{tabular}{|c|c|c|c|c|}
\hline Frequency & Impedance(s11) & Q & Coupl.Coeff. & $\mathrm{d}$ \\
\hline 352.27 & $49.87-\mathrm{j} 1.5$ & 9450 & 1.116 & 1.3 \\
352.31 & $49.9+\mathrm{j} .1$ & 6950 & 1.878 & 1.0 \\
352.33 & $49.1+\mathrm{j} .82$ & 5900 & 2.390 & 0.8 \\
352.30 & $50.5+\mathrm{j} .9$ & 5300 & 2.774 & 0.72 \\
352.36 & $50.3+\mathrm{j} .9$ & 4500 & 3.444 & 0.55 \\
352.27 & $50.08+\mathrm{j} .2$ & 4050 & 3.938 & 0.5 \\
352.27 & $50.3+\mathrm{j} .45$ & 4000 & 4.0 & 0.5 \\
352.33 & $50.5+\mathrm{j} 1.2$ & 3200 & 5.25 & 0.3 \\
352.32 & $49.8+\mathrm{j} .3$ & 3000 & 5.667 & 0.2 \\
\hline
\end{tabular}




\section{Conclusion}

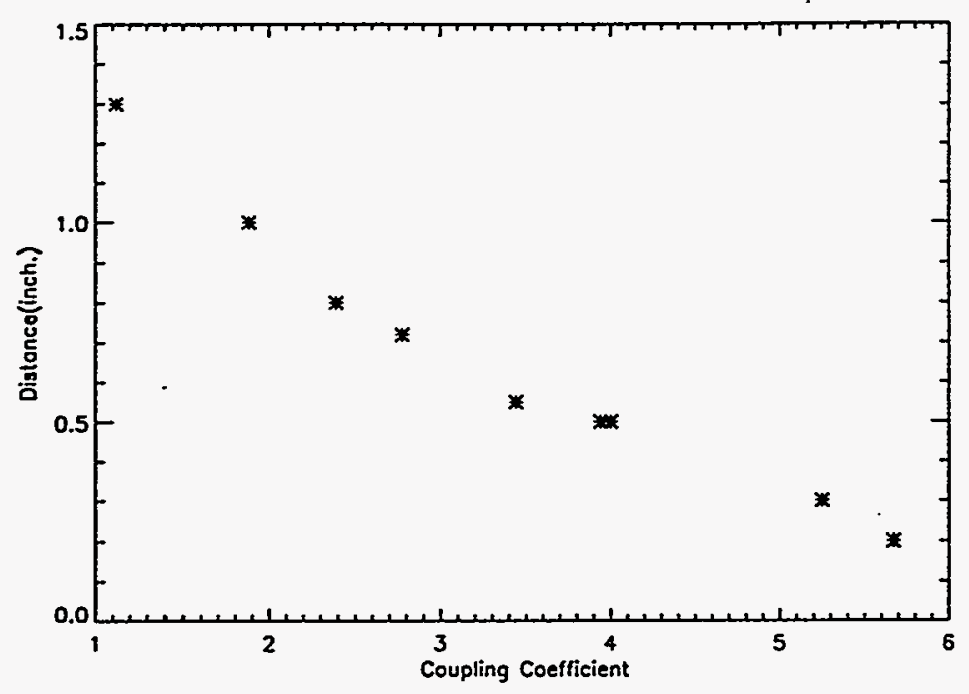

Figure 6: Coupling Coefficient vs. Gap Width

The results obtained in the final test agreed with the preliminary test result in that a negative correlation was shown between the coupling coefficient and the distance. The actual maximum beam loading in the storage ring single-cell cavities will be around $\beta=4$. Therefore, the input coupler will have to move about 0.8 inches inward in order to achieve matching when the maximum beam current is stored in the ring.

\section{References}

1. 1.3.1.4 Storage Ring RF Systems, APS RF Design Handbook.

2. Ginzton, Edward, Microwave Measurements, McGraẉ-Hill, 1957. 